\title{
Inflammatory arthritis and sicca syndrome induced by nivolumab and ipilimumab
}

\author{
Laura C Cappelli, ${ }^{1}$ Anna Kristina Gutierrez, ${ }^{1}$ Alan N Baer, ${ }^{1}$ Jemima Albayda, ${ }^{1}$ \\ Rebecca L Manno, ${ }^{1}$ Uzma Haque, ${ }^{1}$ Evan J Lipson, ${ }^{2}$ Karen B Bleich, ${ }^{3}$ Ami A Shah, ${ }^{1}$ \\ Jarushka Naidoo, ${ }^{2}$ Julie R Brahmer, ${ }^{2}$ Dung Le, ${ }^{2}$ Clifton 0 Bingham III
}

\begin{abstract}
Handling editor Tore K Kvien
${ }^{1}$ Division of Rheumatology, Department of Medicine, Johns Hopkins University, Baltimore, Maryland, USA

${ }^{2}$ Department of Oncology, Johns Hopkins University, Baltimore, Maryland, USA ${ }^{3}$ Department of Radiology, Johns Hopkins University, Baltimore, Maryland, USA
\end{abstract}

\section{Correspondence to} Dr Laura C Cappelli, Division of Rheumatology, Department of Medicine, Johns Hopkins University, 5200 Eastern Avenue, MFL Center Tower 4100, Baltimore, MD 21224, USA; Icappel1@jhmi.edu

Received 22 March 2016 Revised 16 May 2016 Accepted 27 May 2016 Published Online First 15 June 2016

\footnotetext{
To cite: Cappelli LC, Gutierrez AK, Baer AN et al. Ann Rheum Dis 2017;76:43-50.
}

ABSTRACT

Objectives Immune checkpoint inhibitors (ICls) targeting the cytotoxic T-lymphocyte-associated protein 4 (CTLA-4) and programmed cell death protein 1 (PD-1) pathways have demonstrated survival improvements in multiple advanced cancers, but also cause immunerelated adverse events (IRAEs). IRAEs with clinical features similar to rheumatic diseases have not been well described. We report patients with inflammatory arthritis and sicca syndrome secondary to $\mathrm{ICls}$.

Methods We report patients evaluated in the Johns Hopkins Rheumatology clinics from 2012 to 2016 identified as having new rheumatological symptoms in the context of treatment with ipilimumab (anti-CTLA-4) and/or nivolumab (anti-PD-1) for solid tumours.

Results We identified 13 patients who received ICls and developed rheumatological IRAEs. Mean age was 58.7 years. Cancer types included melanoma, non-small cell lung cancer, small cell lung cancer and renal cell carcinoma. $\mathrm{ICl}$ regimens included nivolumab or ipilimumab as monotherapy $(n=5)$, or combination nivolumab and ipilimumab $(n=8)$. Nine of 13 patients developed an inflammatory arthritis, 4 with synovitis confirmed on imaging ( 3 ultrasound, $1 \mathrm{MRI}$ ) and 4 with inflammatory synovial fluid. Four patients developed sicca syndrome with severe salivary hypofunction. Other IRAEs included: pneumonitis, colitis, interstitial nephritis and thyroiditis. Antinuclear antibodies were positive in 5 out of 13 patients. All 13 patients were treated with corticosteroids with varying response. Two patients were treated with methotrexate and antitumor necrosis factor therapy for inflammatory arthritis.

Conclusions As ICls are increasingly used for a range of malignancies, new cases of rheumatic IRAEs are likely to emerge. Further research is required to understand mechanisms, determine risk factors and develop management algorithms for rheumatic IRAEs.

\section{INTRODUCTION}

In recent years, harnessing the power of a patient's immune system to treat cancer has been an increasingly efficacious treatment strategy in oncology. ${ }^{1}$ Immune checkpoint inhibitors (ICI) targeting cytotoxic T-lymphocyte-associated protein 4 (CTLA-4), specifically ipilimumab, and programmed cell death protein 1 (PD-1), nivolumab and pembrolizumab, and the combination of ipilimumab with nivolumab have been Food and Drug Administration (FDA)-approved for the treatment of metastatic melanoma. ${ }^{2-4}$ In the European Union, ipilimumab was approved for metastatic melanoma in $2013,{ }^{5}$ with pembrolizumab and nivolumab approved within the past year. Nivolumab and pembrolizumab are also FDA-approved for metastatic nonsmall cell lung cancer (NSCLC) in the second-line setting and for programmed death ligand 1 (PDL-1)-positive NSCLCs, ${ }^{6}$ and nivolumab has approval for the treatment of renal cell carcinoma (RCC). ${ }^{7}$ In addition, these and other agents targeting related immune pathways, including PDL-1, T-cell immunoglobulin and mucin domain 3 (TIM-3) and lymphocyte activation gene 3 (LAG-3), are currently being tested in a variety of cancers, from solid tumours to haematological malignancies. $^{8} 9$ Hundreds of trials of ICIs are ongoing in the USA. ${ }^{10}$ Although ICI use continues to increase, consequences of these therapies as a result of inducing autoimmunity or through other mechanisms are only beginning to be understood.

A range of immune-related adverse events (IRAEs) have been described with these agents, ranging from severe and potentially life-threatening pneumonitis and colitis, to autoimmune thyroid disease, hypophysitis and vitiligo. ${ }^{11}$ Rates of events have differed by drug and tumour type. ${ }^{11}{ }^{12}$ Rheumatic and musculoskeletal IRAEs have to date not been widely recognised or well characterised. Recognising the potential for ICIs to cause IRAEs that resemble more classical autoimmune diseases will become increasingly important to rheumatologists as more patients are referred for evaluation and management, and to oncologists who must recognise these toxicities in order to refer.

In this article, we report a series of patients evaluated in the Johns Hopkins Rheumatology outpatient clinics from 2012 to 2016 with inflammatory arthritis or sicca symptoms that occurred after the administration of ICIs. We report the clinical, autoantibody, radiological and functional features of these patients, including the oncological and rheumatological treatment they received, and their clinical course and outcomes.

\section{PATIENTS AND METHODS}

All patients were 18 years or older and treated for a malignancy with ipilimumab and/or nivolumab at the Sidney Kimmel Cancer Center at the Johns Hopkins Medical Institutions from 2012 to 2016 for metastatic melanoma, NSCLC or RCC. Patients were referred by oncologists when they identified new clinically important symptoms in routine care 
of patients in practice or trials. Cases were included only if rheumatic signs or symptoms developed after receiving therapy with ipilimumab and/or nivolumab, in the absence of known antecedent inflammatory arthritis or sicca syndrome. Patients underwent a comprehensive rheumatological assessment by a rheumatologist and were classified as having inflammatory arthritis based on history, examination and imaging findings as determined by the treating rheumatologist. Sicca syndrome was defined by the presence of severe salivary hypofunction on examination or functional assessment of salivary flow and/or severe dry eyes as determined by an ophthalmologist.

Demographic data, other IRAE manifestations, treatment of IRAEs and response to therapy and articular findings were recorded by the examining rheumatologists and abstracted from medical records. Cancer treatment responses were defined by Response Evaluation Criteria In Solid Tumors (RECIST) 1.1 criteria $^{13}$ as read by a radiologist on serial CT imaging. The reported laboratory studies are those obtained during the course of clinical care in clinical laboratories. Any imaging studies available in the Johns Hopkins system were also included. In addition, musculoskeletal ultrasound was performed on select patients by an ultrasound-certified rheumatologist.

\section{RESULTS}

\section{Demographics and oncological history}

Six patients had melanoma, five had NSCLC, one had small cell lung carcinoma and one had RCC. The average age of patients was 58.7 years (SD 12), and $83 \%$ were male. Eight of 13 patients received combination therapy with nivolumab and ipilimumab; the remaining five patients received monotherapy with either nivolumab or ipilimumab (table 1). Seven patients had a partial response, five patients had stable disease and one had progressive disease (table 1 ). The median time to develop the first IRAE after exposure to ICIs was 3 months (range: 1-18 months) (table 2).

\section{Inflammatory arthritis}

Nine of the 13 patients had inflammatory arthritis. Clinical features of their musculoskeletal manifestations are described in table 3 .

The clinical presentation was variable between patients and involved both large and small joints in the upper and lower extremities. The initial joints involved were large ones for some patients, especially knees and elbows, while others had initial involvement of the metacarpophalangeal (MCP) and proximal interphalangeal (PIP) joints. When followed over time, six patients evolved to develop rheumatoid arthritis (RA)-like pattern characterised by bilateral synovitis of the PIPs, MCPs and/or wrists, along with larger joint involvement. Five of these patients were treated with systemic corticosteroids. Interestingly, most of these patients had profound inflammatory disease requiring much higher doses of corticosteroid therapy than initially anticipated based on their clinical phenotype. Also, the inflammatory arthritis persisted in some patients for months after ICI therapy was stopped. Patient 1 still had active inflammatory arthritis 6 months after his last infusion of nivolumab, and patient 2 had active inflammatory arthritis 15 months after her last infusion of ipilimumab and nivolumab. In these two patients, therapy was intensified to include tumour necrosis factor (TNF)-inhibitors, ultimately resulting in adequate control of their arthritis.

Two patients had a clinical presentation similar to reactive arthritis. One of these individuals (patient 6) had dactylitis of one finger and large joint oligoarthritis with conjunctivitis and urethritis. His symptoms resolved when he was treated with infliximab and prednisone for colitis and did not recur. The other (patient 4) had urethritis and conjunctivitis followed closely by a predominantly large joint polyarthritis. He required doses of corticosteroids $>1 \mathrm{mg} / \mathrm{kg}$ for 2 weeks before having any improvement in his articular manifestations. Adalimumab was initiated with considerable improvement allowing tapering of steroids.

One patient had a large joint polyarthritis consistent with a seronegative spondyloarthritis (patient 7). He did not have synovitis of the PIPs or MCPs, back pain, enthesitis or extraarticular manifestations of reactive arthritis. He responded to $40 \mathrm{mg}$ daily of prednisone therapy with symptoms recurring when he decreased below $10 \mathrm{mg}$ daily.

Imaging was conducted in four patients and confirmed the presence of inflammatory arthritis in each. Three had abnormal musculoskeletal ultrasound findings, and one had an abnormal joint MRI. Ultrasound demonstrated synovitis with erosions in the right talonavicular joint of a patient with seronegative symmetric polyarthritis (patient 2) similar to RA (figure 1). In patient 1, MRI showed tibiotalar and subtalar joint effusions (figure 2). Patient 3 had erosive arthritis and proliferative synovitis of the elbow by ultrasound at the time of his initial rheumatological evaluation (table 2). Initial plain films were negative for metastatic bone lesions, but he ultimately developed a metastatic lesion in the humerus, just proximal to the elbow.

Four patients had synovial fluid analysis, each inflammatory (synovial fluid white blood cells range 9854-28 400 cells $/ \mathrm{mm}^{3}$ ). All synovial fluids had a predominance of polymorphonuclear cells $(>70 \%)$. Patient 7 had synovial fluid monosodium urate crystals, with a synovial fluid white blood cell count of 9854 cells $/ \mathrm{mm}^{3}$ and a serum uric acid level of $5.7 \mathrm{mg} / \mathrm{dL}$. However, his presentation was thought to be more indicative of a non-crystalline disease, given his failure to respond to colchicine, his polyarticular presentation and his ongoing requirement for high doses of corticosteroids (up to $40 \mathrm{mg}$ /day prednisone).

No patients with inflammatory arthritis were positive for rheumatoid factor (RF) or anti-cyclic citrullinated peptide (CCP) antibodies. Three of the inflammatory arthritis patients had antinuclear antibodies (ANA), only one with a high titre (table 2).

Four of the nine patients with inflammatory arthritis also developed colitis during their ICI treatment. For three patients, the colitis preceded the arthritis, and in the fourth the colitis developed after the arthritis. Two of these patients also had reactive arthritis presentations, and one had symptoms suggestive of inflammatory back pain.

\section{Sicca syndrome}

Four patients had sicca symptoms that developed while on ICIs and could not be explained by other medications. All four presented with the relatively abrupt onset of severe dry mouth symptoms and had examination evidence of severe salivary hypofunction. One patient had concurrent bilateral parotid gland swelling which resolved with steroid therapy. On ultrasound imaging, she also had discrete hypoechoic foci occupying more than $50 \%$ of her parotid and submandibular glands, changes that are commonly seen in Sjögren's syndrome. ${ }^{14}$ One patient had a pre-existing history of severe dry eye disease, but no diagnosis of Sjögren's syndrome and no severe dry mouth symptoms. Two of the patients with sicca syndrome developed other IRAEs; pneumonitis developed in one patient and interstitial nephritis and colitis in the other. The pneumonitis, interstitial nephritis and colitis improved with steroid treatment. 


\begin{tabular}{|c|c|c|c|c|c|c|c|}
\hline Patient & Age & Sex & Race & Type of malignancy & Cancer therapy & Rheumatic IRAE & $\begin{array}{l}\text { Best overall response } \\
\text { (RECIST 1.1) }\end{array}$ \\
\hline 1 & 58 & Male & Caucasian & Renal cell carcinoma & $\begin{array}{l}\text { Anti-PD-1 } \\
\text { Anti-CTLA-4 }\end{array}$ & Inflammatory arthritis & Stable disease \\
\hline 2 & 46 & Female & Caucasian & Melanoma & $\begin{array}{l}\text { Anti-PD-1 } \\
\text { Anti-CTLA-4 }\end{array}$ & Inflammatory arthritis & Partial response \\
\hline 3 & 62 & Male & African American & Non-small cell lung cancer & $\begin{array}{l}\text { Anti-PD-1 } \\
\text { Anti-CTLA-4 }\end{array}$ & Inflammatory arthritis & Stable disease \\
\hline 4 & 35 & Male & Caucasian & Melanoma & $\begin{array}{l}\text { Anti-PD-1 } \\
\text { Anti-CTLA-4 }\end{array}$ & Inflammatory arthritis & Stable disease \\
\hline 5 & 56 & Male & Caucasian & Non-small cell lung cancer & Anti-PD-1 & Inflammatory arthritis & Stable disease \\
\hline 6 & 66 & Male & Caucasian & Melanoma & $\begin{array}{l}\text { Anti-PD-1 } \\
\text { Anti-CTLA-4 }\end{array}$ & Inflammatory arthritis & Partial response \\
\hline 7 & 57 & Male & Caucasian & Small cell lung cancer & $\begin{array}{l}\text { Anti-PD-1 } \\
\text { Anti-CTLA-4 }\end{array}$ & Inflammatory arthritis & Partial response \\
\hline 8 & 42 & Male & Caucasian & Non-small cell lung cancer & $\begin{array}{l}\text { Anti-PD-1 } \\
\text { Anti-CTLA-4 }\end{array}$ & Inflammatory arthritis & Partial response \\
\hline 9 & 75 & Female & Caucasian & Non-small cell lung cancer & Anti-PD-1 & Inflammatory arthritis & Partial response \\
\hline 10 & 61 & Male & Caucasian & Non-small cell lung cancer & Anti-PD-1 & Sicca syndrome & Stable disease \\
\hline 11 & 57 & Male & Caucasian & Melanoma & $\begin{array}{l}\text { Anti-PD-1 } \\
\text { Anti-CTLA-4 }\end{array}$ & Sicca syndrome & Progressive disease \\
\hline 12 & 74 & Male & Caucasian & Melanoma & Anti-CTLA-4 & Sicca syndrome & Partial response \\
\hline 13 & 74 & Female & Caucasian & Melanoma & Anti-PD-1 & Sicca syndrome & $\begin{array}{l}\text { No measureable disease } \\
\text { by RECIST; tumour regression } \\
\text { observed on clinical exam }\end{array}$ \\
\hline
\end{tabular}

Age is at the time of initial rheumatological assessment.

CTLA-4, cytotoxic T-lymphocyte-associated protein 4; PD-1, programmed cell death protein 1.

Three of four sicca patients had positive ANA; one patient had low titre La/SSB antibodies; but none of the patients had Ro/SSA antibodies (table 2). One sicca patient had a positive RF. One patient, who also had pneumonitis, had anti-EJ antibodies (one of the antisynthetase antibodies). He had no associated rash, Raynaud's phenomenon or proximal muscle weakness.

\section{DISCUSSION}

A range of IRAEs in the setting of ICIs have been recognised and well described, including algorithms for their management. Less severe IRAEs, such as dermatological IRAEs, can occur in $>30 \%$ of patients who received nivolumab or pembrolizumab for melanoma. ${ }^{11}$ Hypothyroidism, also common, occurs in as many as $15 \%$ of patients treated with concurrent nivolumab and ipilimumab therapy for melanoma ${ }^{4}$ versus $6 \%$ in those patients treated with ipilimumab alone. ${ }^{5}$ More severe events like colitis, pneumonitis and hepatitis are less common. In patients with melanoma treated with ipilimumab, severe immune-related colitis has been described in approximately $5 \%$ of patients, ${ }^{4} 5$ whereas rates of colitis were only about $1 \%$ in phase III studies of nivolumab for metastatic melanoma ${ }^{2}$ and NSCLC. ${ }^{4}$ Combination therapy with ipilimumab and nivolumab has shown higher rates of colitis, $11.8 \%$ in one trial. ${ }^{4}$ Pneumonitis has been seen in $5 \%$ of patients treated with nivolumab for squamous NSCLC, ${ }^{15} 4 \%$ in those treated for $\mathrm{RCC}^{7}$ and $1.5 \%$ in melanoma. ${ }^{16}$ Autoimmune hepatitis is reported in $5 \%-10 \%$ of patients treated with nivolumab or ipilimumab. ${ }^{16-19}$ Inflammatory conditions affecting the skin, eye, kidney, pancreas and neurological system have also been described. ${ }^{20}$

Inflammatory arthritis and sicca syndrome associated with ICI use have been less commonly reported. Although arthralgia has been noted (in 5\%-16\% of patients treated with nivolumab in phase III studies for melanoma ${ }^{2}$ or NSCLC $^{21}$ and in $11 \%$ of those treated with combination ipilimumab/nivolumab therapy, ${ }^{22}$ inflammatory arthritis is less commonly described. The current literature consists of case reports or small case series. Two cases of polyarticular seronegative inflammatory arthritis developing after pembrolizumab therapy were recently reported. ${ }^{23}$ One patient had a large joint arthritis, while the other had both large and small (PIP) joint involvement. Both patients lacked RF, CCP and ANA. In a retrospective review of imaging studies using CT and positron emission tomography (PET)/CT performed before and after treatment with antiCTLA-4 therapies, 4 of 119 patients had evidence of new arthritis detectable on imaging. ${ }^{24}$ Limited clinical information was reported on these patients to further characterise them.

This report provides the largest case series to date of inflammatory arthritis due to ICIs. This series illustrates the wide range of clinical presentations and management required for patients with rheumatic IRAEs. We describe clinically relevant subtypes of inflammatory arthritis, which may involve both large and small joints, and present as an oligoarthritis, an additive arthritis or with severe polyarthritis. The arthritis can be severe and erosive, requiring multiple immunosuppressant medications, or, less frequently, can be mild with improvement on low-dose corticosteroids or non-steroidal anti-inflammatory drugs alone. We observed that the inflammatory arthritis may also persist after ICI discontinuation. Though many patients had symmetrical small joint involvement consistent with RA, anti-CCP and RF were not seen in most patients with ICI-induced arthritis. In addition, the dose of steroids required to control symptoms was often higher than would be typically adequate to manage other forms of inflammatory arthritis. The presence of rapid erosive and destructive disease should raise concerns of potential metastatic disease, as seen in one of the patients reported here. The large joint involvement in most patients, along with the reactive arthritis phenotype and 
Table 2 Autoimmune disease phenotype, antibodies and treatment for included patients

\begin{tabular}{|c|c|c|c|c|c|c|c|}
\hline & $\begin{array}{l}\text { Date of first } \\
\text { ICI } \\
\text { exposure }\end{array}$ & $\begin{array}{l}\text { Autoimmune disease } \\
\text { phenotype }\end{array}$ & Date of IRAE & Treatment of IRAE & IRAE response to treatment & Imaging, synovial fluid and other findings & Autoantibody results \\
\hline \multirow[t]{2}{*}{1} & \multirow[t]{2}{*}{$21 / 2 / 2014$} & & $21 / 3 / 2014$ & $\begin{array}{l}\text { Prednisone } 120 \mathrm{mg} \text { daily, tapered off over } \\
3 \text { months. ICI held for } 3 \text { months }\end{array}$ & $\begin{array}{l}\text { Resolution of colitis. Able to go back } \\
\text { on ICI }\end{array}$ & & \multirow[b]{2}{*}{$\begin{array}{l}\text { ANA, RF, CCP, Ro, La, dsDNA, } \\
\text { RNP, Sm, myositis panel**, Scl70, } \\
\text { HLA-B27 negative }\end{array}$} \\
\hline & & Inflammatory arthritis & $\begin{array}{l}\text { 2/2015: reported } \\
\text { 16/6/2015: seen }\end{array}$ & $\begin{array}{l}\text { Prednisone } 10 \mathrm{mg} \text { daily with suboptimal } \\
\text { response. ICI stopped and prednisone } \\
\text { increased to } 40 \mathrm{mg} \text { daily. Continued } \\
\text { disease activity, so MTX } 15 \mathrm{mg} \text { weekly and } \\
\text { adalimumab weekly added to regimen with } \\
\text { improvement }\end{array}$ & $\begin{array}{l}\text { Improvement with adalimumab and } \\
\text { MTX. Able to come off prednisone }\end{array}$ & $\begin{array}{l}\text { MRI: tibiotalar and subtalar joint effusions } \\
\text { with marked synovitis }\end{array}$ & \\
\hline \multirow[t]{4}{*}{2} & \multirow[t]{4}{*}{$7 / 2013$} & Colitis (on anti-CTLA-4) & $10 / 2013$ & Prednisone $1 \mathrm{mg} / \mathrm{kg} / \mathrm{day}$ & Resolution of colitis & & \\
\hline & & Thyroiditis/hypothyroid & $10 / 2013$ & $\begin{array}{l}\text { Prednisone } 40 \mathrm{mg} \text {, tapered to } 7 \mathrm{mg} / \mathrm{day} \\
\text { MTX }\end{array}$ & $\begin{array}{l}\text { Poor response to steroids ( } 4 \text { months) } \\
\text { Poor response to MTX ( } 5 \text { months) }\end{array}$ & \multirow{3}{*}{$\begin{array}{l}\text { Ultrasound: Doppler-positive synovitis in the } \\
\text { right ankle }\end{array}$} & \multirow{3}{*}{ RF, CCP negative } \\
\hline & & $\begin{array}{l}\text { Inflammatory arthritis } \\
\text { (on anti-PD-1) }\end{array}$ & $8 / 2014$ & & $\begin{array}{l}\text { Initial response to infliximab, d/c due } \\
\text { to } A E \text { (nausea, chills) }\end{array}$ & & \\
\hline & & & & Etanercept & Marked response to etanercept & & \\
\hline 3 & $14 / 11 / 2014$ & Inflammatory arthritis & $\begin{array}{l}\text { 3/8/2015- } \\
\text { reported } \\
17 / 9 / 2015 \text {-seen }\end{array}$ & $\begin{array}{l}\text { Intra-articular triamcinolone } \\
\text { Prednisone } 40 \mathrm{mg} \text { daily, tapered off over } \\
1 \text { month. ICl stopped, resumed } 40 \mathrm{mg} \\
\text { prednisone }\end{array}$ & $\begin{array}{l}\text { Temporary relief from intra-articular } \\
\text { triamcinolone, partial relief from } \\
40 \mathrm{mg} \text { prednisone but not smaller } \\
\text { doses }\end{array}$ & $\begin{array}{l}\text { Ultrasound: Doppler-positive synovitis and } \\
\text { erosions at elbow } \\
\text { Subsequently developed metastatic lesion at } \\
\text { distal humerus }\end{array}$ & $\begin{array}{l}\text { ANA, RF, CCP, Ro, La, RNP, Sm, } \\
\text { dsDNA, P-ANCA, C-ANCA, } \\
\text { HLA-B27, ScI70, RNA pol III, } \\
\text { cardiolipin, } \beta \text {-2-glycoprotein } \\
\text { antibodies negative }\end{array}$ \\
\hline \multirow[t]{2}{*}{4} & \multirow[t]{2}{*}{$21 / 10 / 2015$} & & $11 / 2015$ & Prednisone starting at $160 \mathrm{mg}$ daily & Resolved & & \multirow[b]{2}{*}{$\begin{array}{l}\text { ANA, RF, CCP, Ro, La, RNP, Sm, } \\
\text { dsDNA, P-ANCA, C-ANCA, } \\
\text { HLA-B27, Scl70, cardiolipin } \\
\text { antibodies, } \beta \text {-2-glycoprotein } \\
\text { antibodies negative }\end{array}$} \\
\hline & & Inflammatory arthritis & $1 / 2016-$ & $\begin{array}{l}\text { Prednisone } 120 \mathrm{mg} \text { daily tapered down to } \\
40 \mathrm{mg} \text { daily } \\
\text { Intra-articular triamcinolone } \\
\text { Adalimumab initiated }\end{array}$ & $\begin{array}{l}\text { Initial steroid treatment with no } \\
\text { improvement, after } 2 \text { weeks high-dose } \\
\text { steroids some improvement in pain } \\
\text { and swelling. Marked improvement } \\
\text { with adalimumab allowing steroid } \\
\text { taper }\end{array}$ & Synovial fluid: 11950 WBCs (92\% PMN) & \\
\hline \multirow[t]{2}{*}{5} & \multirow[t]{2}{*}{$26 / 3 / 2015$} & \multirow[t]{2}{*}{ Inflammatory arthritis } & $\begin{array}{l}21 / 5 / 2015- \\
\text { reported } \\
25 / 6 / 2015 \text {-seen }\end{array}$ & $\begin{array}{l}\text { Prednisone } 10 \mathrm{mg} \text { daily, tapered to } 7.5 \mathrm{mg} \\
\text { daily after } 3 \text { months }\end{array}$ & \multirow[t]{2}{*}{$\begin{array}{l}\text { Good response with improvement of } \\
\text { symptoms }\end{array}$} & \multirow[t]{2}{*}{ None available } & $\begin{array}{l}\text { ANA 1:80 (speckled) } \\
\text { Anti-Ro positive }\end{array}$ \\
\hline & & & & & & & $\begin{array}{l}\mathrm{RF}, \mathrm{CCP}, \mathrm{RNP}, \mathrm{Sm}, \mathrm{Scl} 70 \text {, myositis } \\
\text { panel negative }\end{array}$ \\
\hline \multirow[t]{7}{*}{6} & \multirow[t]{7}{*}{$3 / 2012$} & \multirow[t]{5}{*}{ Colitis } & $5 / 2013$ & $\begin{array}{l}\text { Prednisone } 80 \mathrm{mg} / \text { day tapered to } 20 \mathrm{mg} / \\
\text { day }\end{array}$ & $\begin{array}{l}\text { Initial response high dose steroids, } \\
\text { recurrence with steroid taper } \\
\text { Resolved with infliximab }\end{array}$ & \multirow{3}{*}{$\begin{array}{l}\text { Colonoscopy: mild, left-sided colitis } \\
\text { (descending colon, sigmoid colon and rectum } \\
\text { notable for mild erythema } \\
\text { without ulceration) }\end{array}$} & \\
\hline & & & $5 / 2 / 2014$ & Infliximab (one dose) & & & \\
\hline & & & $\begin{array}{l}\text { (recurrence after } \\
\text { re-dosing with ICI }\end{array}$ & & $\begin{array}{l}\text { Poor response to high dose steroids } \\
\text { Adverse event (pneumonia) }\end{array}$ & & \\
\hline & & & 1 month prior) & Infliximab (two doses) & Resolved & \multirow{2}{*}{$\begin{array}{l}\text { Knee radiographs: large suprapatellar } \\
\text { effusion, no erosions }\end{array}$} & \\
\hline & & & 20/12/2013 & & & & \\
\hline & & \multirow{2}{*}{$\begin{array}{l}\text { Hypothyroidism } \\
\text { Reactive arthritis } \\
\text { (inflammatory arthritis, } \\
\text { conjunctivitis) }\end{array}$} & 5/1/2014-seen & $\begin{array}{l}\text { Prednisone } 40 \mathrm{mg} / \mathrm{day} \text { tapered over } \\
1 \text { months to } 10 \mathrm{mg} / \mathrm{day}\end{array}$ & $\begin{array}{l}\text { stiffness at } 10 \mathrm{mg} / \text { day } \\
\text { Improved with steroid injection }\end{array}$ & \multirow[t]{2}{*}{$\begin{array}{l}\text { Synovial fluid analysis: WBC } 28455 \\
\text { (70\% PMNs) }\end{array}$} & \multirow[t]{2}{*}{$\begin{array}{l}\text { ANA, RF, CCP, antihistone } \\
\text { negative }\end{array}$} \\
\hline & & & $\begin{array}{l}7 / 3 / 2014 \text { (flare } \\
\text { after infliximab) }\end{array}$ & $\begin{array}{l}\text { Intra-articular triamcinolone } \\
\text { Prednisone } 1 \mathrm{mg} / \mathrm{kg} / \mathrm{day} \\
\text { Intra-articular triamcinolone }\end{array}$ & $\begin{array}{l}\text { Improved with steroids } \\
\text { Improved with adalimumab }\end{array}$ & & \\
\hline
\end{tabular}




\begin{tabular}{|c|c|c|c|c|c|c|c|}
\hline & $\begin{array}{l}\text { Date of first } \\
\mathrm{ICI} \\
\text { exposure }\end{array}$ & $\begin{array}{l}\text { Autoimmune disease } \\
\text { phenotype }\end{array}$ & Date of IRAE & Treatment of IRAE & IRAE response to treatment & Imaging, synovial fluid and other findings & Autoantibody results \\
\hline 7 & 3/3/2015 & $\begin{array}{l}\text { Inflammatory arthritis } \\
\text { Thyroiditis/ } \\
\text { hypothyroidism }\end{array}$ & $\begin{array}{l}\text { 19/11/2015- } \\
\text { reported } \\
7 / 12 / 15 \text {-seen } \\
\text { 17/4/2015 }\end{array}$ & $\begin{array}{l}\text { Prednisone } 10 \mathrm{mg} \text { daily } \\
\text { Intra-articular triamcinolone } \\
\text { Colchicine } 0.6 \mathrm{mg} \text { daily } \\
\text { Propranolol initially, Levothyroxine for } \\
\text { subsequent hypothyroidism }\end{array}$ & $\begin{array}{l}\text { Improvement of symptoms on } 10 \mathrm{mg} \\
\text { prednisone, but progressive symptoms } \\
\text { involving the shoulder, knee, wrist } \\
\text { and elbow requiring } 40 \mathrm{mg} \\
\text { prednisone for relief }\end{array}$ & $\begin{array}{l}\text { Ultrasound: Synovitis with positive Doppler } \\
\text { signal in knee, elbow } \\
\text { Synovial fluid: } 9854 \text { WBC ( } 86 \% \text { PMN, } 14 \% \\
\text { mononuclear, monosodium urate crystals } \\
\text { present) }\end{array}$ & $\begin{array}{l}\text { ANA, RF, CCP, Ro, La, RNP, Sm, } \\
\text { dsDNA, P-ANCA, C-ANCA, } \\
\text { HLA-B27, Scl70, RNA pol III, } \\
\text { cardiolipin antibodies, } \\
\beta \text {-2-glycoprotein antibodies } \\
\text { negative }\end{array}$ \\
\hline 8 & $26 / 2 / 2015$ & Inflammatory arthritis & $\begin{array}{l}\text { 5/2015-reported } \\
\text { 9/12/2015-seen }\end{array}$ & $\begin{array}{l}\text { Dexamethasone } 8 \mathrm{mg} \text { daily for brain } \\
\text { metastasis, no additional corticosteroids } \\
\text { added }\end{array}$ & $\begin{array}{l}\text { Improvement of joint symptoms on } \\
\text { dexamethasone }\end{array}$ & None & $\begin{array}{l}\text { ANA, RF, CCP, Ro, La, RNP, Sm, } \\
\text { dsDNA, P-ANCA, C-ANCA, } \\
\text { HLA-B27, Scl70, RNA pol III, } \\
\text { cardiolipin antibodies, } \\
\beta \text {-2-glycoprotein antibodies } \\
\text { negative }\end{array}$ \\
\hline 9 & 16/7/2015 & Inflammatory arthritis & $\begin{array}{l}\text { 12/2015- } \\
\text { reported } \\
\text { 2/2016-seen }\end{array}$ & $\begin{array}{l}\text { Celecoxib twice daily, intra-articular } \\
\text { triamcinolone }\end{array}$ & & None available & $\begin{array}{l}\text { ANA, RF, CCP, Ro, La, RNP, Sm, } \\
\text { dsDNA, P-ANCA, C-ANCA, Scl70, } \\
\text { cardiolipin antibodies, } \\
\beta \text {-2-glycoprotein antibodies } \\
\text { negative }\end{array}$ \\
\hline 10 & $1 / 5 / 2015$ & $\begin{array}{l}\text { Pneumonitis } \\
\text { Acute sicca }\end{array}$ & $\begin{array}{l}\text { 23/7/15 } \\
\text { 9/7/15—reported } \\
\text { 30/7/15—seen }\end{array}$ & $\begin{array}{l}\text { Prednisone } 80 \mathrm{mg} \text { daily, tapered over } \\
2 \text { months } \\
\text { Pilocarpine for symptoms }\end{array}$ & $\begin{array}{l}\text { Improvement of imaging and } \\
\text { symptoms } \\
\text { Improvement of xerostomia }\end{array}$ & None available & $\begin{array}{l}\text { ANA 1:320 (nucleolar) } \\
\text { RF } 38 \text { (ULN 35) } \\
\text { Anti-EJ antibodies positive, rest of } \\
\text { myositis panel negative. } \\
\text { Ro, La, RNP, Sm, Scl70 negative }\end{array}$ \\
\hline 11 & $24 / 4 / 2015$ & Acute sicca & $6 / 2015$ & $\begin{array}{l}\text { Prednisone } 0.5 \mathrm{mg} / \mathrm{kg} / \mathrm{day} \text {, increased to } \\
1 \mathrm{mg} / \mathrm{kg} / \text { day, tapered to until discontinued } \\
\text { Cevimeline for symptoms }\end{array}$ & $\begin{array}{l}\text { No improvement } \\
\text { No benefit }\end{array}$ & & $\begin{array}{l}\text { ANA 1:320 homogenous } \\
\text { Ro, La, RF negative }\end{array}$ \\
\hline 12 & $\begin{array}{l}\text { 6/9/2011 } \\
\text { nivolumab } \\
\text { 8/3/2013 } \\
\text { ipilimumab }\end{array}$ & $\begin{array}{l}\text { Acute sicca } \\
\text { Interstitial nephritis } \\
\text { Insulin-dependent } \\
\text { diabetes } \\
\text { Colitis }\end{array}$ & $\begin{array}{l}1 / 5 / 2012 \\
22 / 5 / 2012 \text {-seen }\end{array}$ & $\begin{array}{l}\text { Prednisone } 1 \mathrm{mg} / \mathrm{kg} / \text { day } \\
\text { Prednisone } 1 \mathrm{mg} / \mathrm{kg} / \text { day } \\
\text { Insulin }\end{array}$ & $\begin{array}{l}\text { Improvement in xerostomia } \\
\text { Improvement in creatinine } \\
\text { Maintained on insulin } \\
\text { Resolved with steroids }\end{array}$ & $\begin{array}{l}\text { Creatinine } 3.1 \\
\text { BUN } 36 \\
\text { Renal biopsy: acute and evolving chronic } \\
\text { interstitial inflammation with some T cells } \\
\text { and focally numerous eosinophils suggesting } \\
\text { a hypersensitivity reaction. The T-cell-rich } \\
\text { lymphocytic infiltrate in the interstitial } \\
\text { kidneys suggested an autoimmune interstitial } \\
\text { nephritis }\end{array}$ & $\begin{array}{l}\text { ANA 1:80 speckled } \\
\text { Ro, La, RF, P-ANCA, C-ANCA } \\
\text { negative }\end{array}$ \\
\hline 13 & 1/4/2015 & $\begin{array}{l}\text { Acute sicca with } \\
\text { parotid swelling }\end{array}$ & 12/2015 & $\begin{array}{l}\text { Prednisone } 40 \mathrm{mg} \text { daily } \\
\text { Cevilemine for symptomatic relief of } \\
\text { xerostomia }\end{array}$ & $\begin{array}{l}\text { Improved parotid swelling, prednisone } \\
\text { tapered off over } 6 \text { weeks }\end{array}$ & $\begin{array}{l}\text { Ultrasound bilateral parotid glands: } \\
\text { hypoechoic foci consistent with lymphocytic } \\
\text { aggregates, }>50 \% \text { of gland involved }\end{array}$ & $\begin{array}{l}\text { La/SSB antibodies positive. ANA, } \\
\text { Ro, RNP, Sm, RF, CCP, myositis } \\
\text { panel, anti-dsDNA, P-ANCA, } \\
\text { C-ANCA negative }\end{array}$ \\
\hline
\end{tabular}

*Myositis panel tests for antibodies to Jo-1, SRP, EJ, OJ, PL-7, PL-12, Mi-2, Ku.

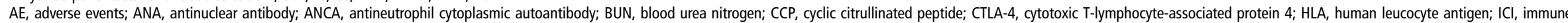
checkpoint inhibitor; MTX, methotrexate; PMN, polymorphonuclear leucocytes; RF, rheumatoid factor; RNP, ribonuclear protein antibody; ULN, upper limit of normal; WBC, white blood cells. 


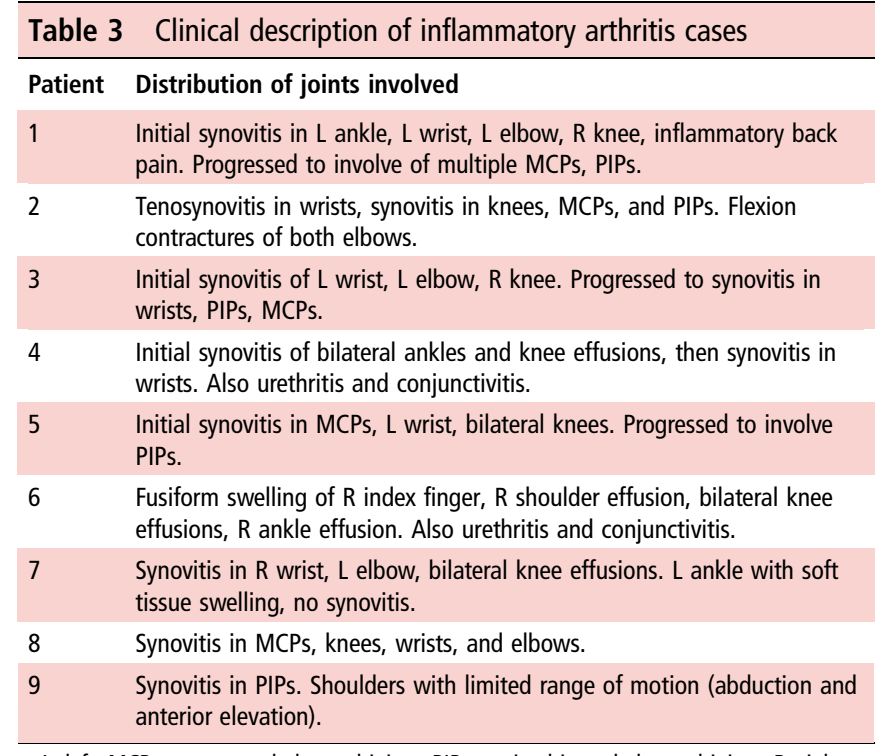

$\mathrm{L}$, left; MCP, metacarpophalangeal joints; PIP, proximal interphalangeal joints; $\mathrm{R}$, right.
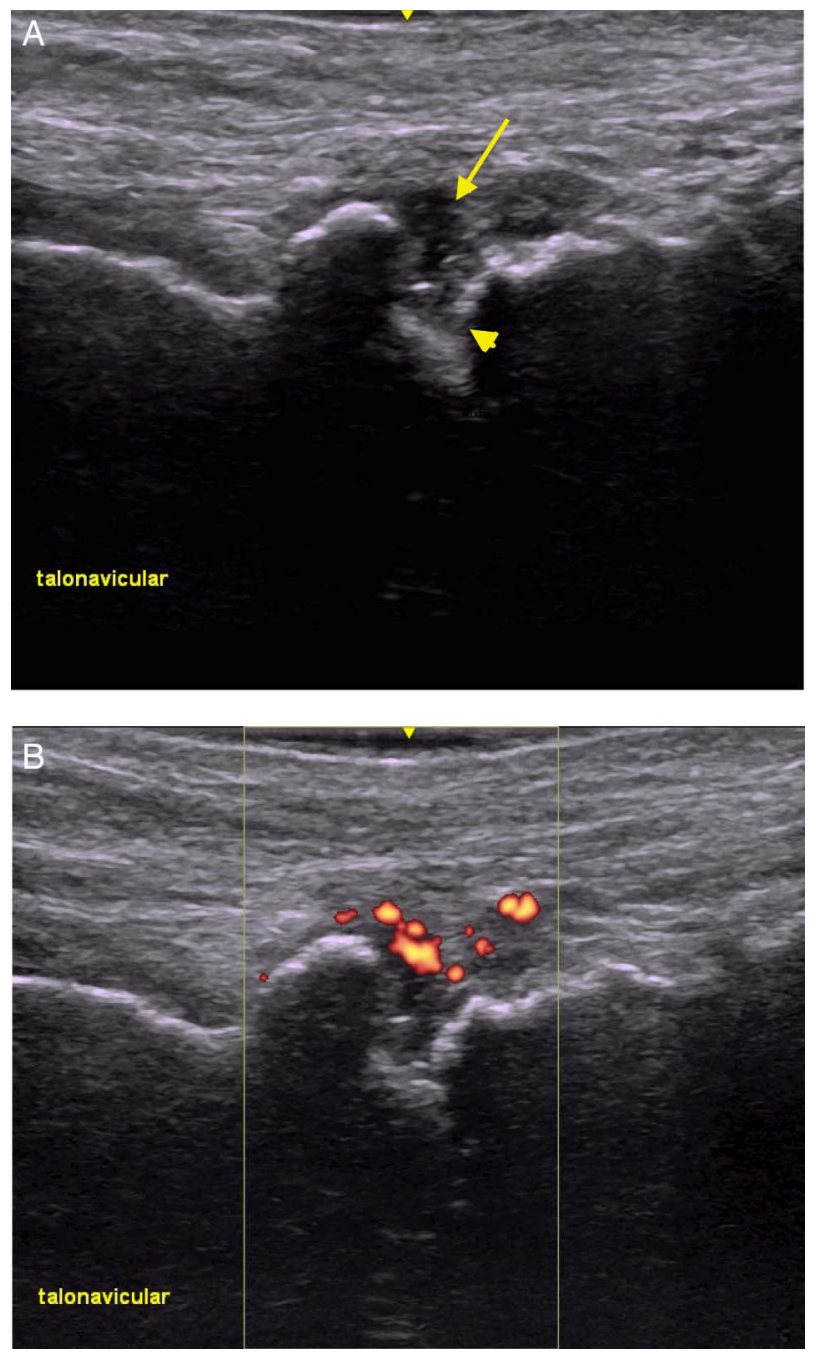

Figure 1 Ultrasound imaging of talonavicular joint showing erosions and synovitis. (A) Grey-scale ultrasound showing a hypoechoic focus (arrow) and erosions (arrowhead). (B) Power Doppler ultrasound showing enhancement of synovitis.

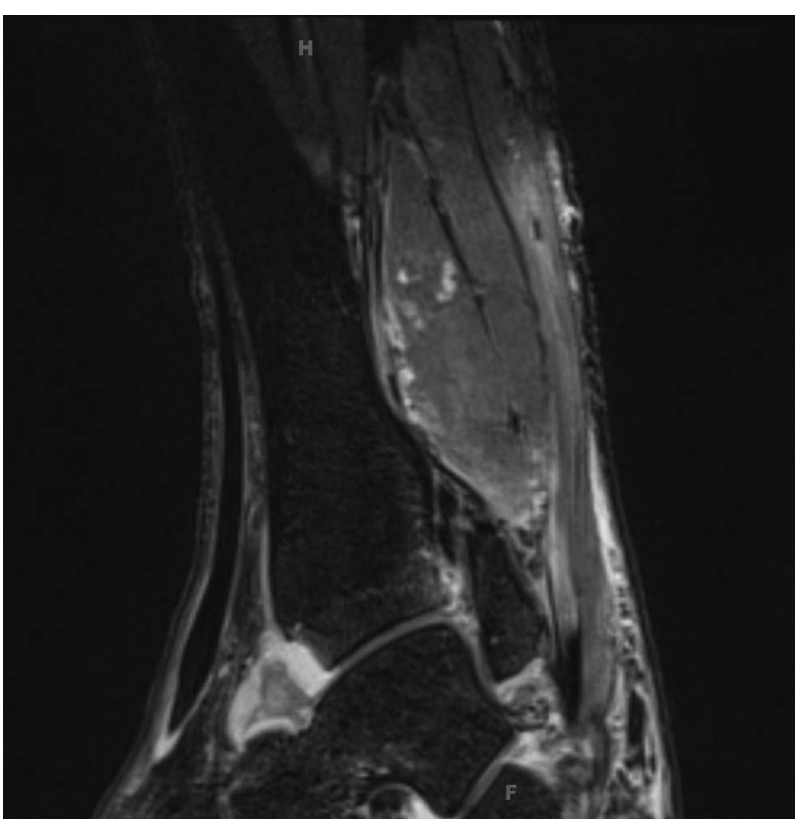

Figure 2 MRI of left ankle. T2 fat-suppressed image showing tibiotalar synovitis.

coexisting colitis, suggest a possible $\mathrm{T}$ helper 17 (Th17)-mediated mechanism of the inflammatory arthritis. Interestingly, PD-1 blockade has been shown to augment the Th17 response in patients with melanoma and prostate cancer. $^{25}$

To our knowledge, no individual cases of ICI-induced sicca syndrome have been previously described. Dry mouth was reported in $6.5 \%$ of patients in one phase I clinical trial of nivolumab, ${ }^{19}$ but has not been reported in larger clinical trials. In the current series, patients with sicca syndrome presented with abrupt and severe salivary hypofunction, suggesting an immune aetiology. The dry eye symptoms were less severe. Ro antibodies were not observed in the four patients with sicca, and parotid gland swelling and La antibodies were seen in one patient.

Although not reported in our series, other IRAEs with rheumatological manifestations have been noted including inflammatory myopathy and vasculitis. One case of ipilimumab-associated dermatomyositis with cutaneous findings, proximal muscle weakness and elevated muscle enzymes was recently published. ${ }^{26}$ Another case of proximal muscle and respiratory weakness, with elevated muscle enzymes following treatment with nivolumab was also reported. ${ }^{27}$ Polymyalgia rheumatica and giant cell arteritis have been reported after treatment with ipilimumab in two patients. $^{28}$

Many of the patients we report had more than one IRAE. In this case series, colitis was the most common concurrent event; pneumonitis and thyroiditis were also seen. The sequence of IRAEs varied; some patients had arthritis or sicca first while others had IRAEs preceding the arthritis or sicca. Also notable was the short duration these subjects were exposed to ICIs before developing their first IRAE. In nearly all (12 of 13 cases), the first IRAE developed after 9 months or less of ICI therapy, with $62 \%$ occurring within the first 3 months and $23 \%$ in the first month following first exposure. The kinetics of the onset of IRAEs has been described for ipilimumab, with skin typically seen as the first manifestation, followed by colitis, and later hepatitis and endocrinopathies. ${ }^{29}$ For the rheumatic manifestations reported here, timing of onset ranged from 2 to 13 months after ICI exposure. 
The true incidence of ICI-induced inflammatory arthritis or sicca syndrome is uncertain as it is difficult to obtain a clear denominator of patients treated with nivolumab and ipilimumab at our institution. The nine patients with inflammatory arthritis seen at our centre come from a pool of both clinical trial patients and patients receiving therapy as standard-of-care. Also, it is unlikely that the numerator is representative of all rheumatic IRAEs experienced for several reasons. Patients may not have been referred to rheumatology if they had mild symptoms, if they had a concurrent IRAE treated with steroids by oncology, if they died from their cancer or if they stopped their ICI for other reasons. A rough estimate of a denominator is about 700 patients treated with immunotherapy in trials at Johns Hopkins, which would give an incidence of $1.3 \%$. We speculate that the incidence of these events will be similar to other previously reported IRAEs when information on arthritis is systematically captured from the beginning of therapy. Current clinical trial data likely underestimates the incidence of inflammatory arthritis due to the numerous options for coding of musculoskeletal symptoms (arthritis, arthralgia, joint effusion) that are mutually exclusive. Prior work has shown that the grading system employed for oncological clinical trials may underestimate symptom severity and impact for musculoskeletal and rheumatic AEs. ${ }^{30}$

Limitations of this study include the retrospective nature of the analysis, and that patients reported here received only nivolumab and/or ipilimumab rather than all currently approved ICIs. The patients included had symptoms of sufficient severity to be referred to a rheumatologist. There may be many patients with milder symptoms of rheumatic IRAEs who were not referred. Our sample of patients was also enriched for participants in clinical trials, as Johns Hopkins is a tertiary referral centre, and nivolumab has been Food and Drug Administration-approved for a short period of time. Patients receiving ICIs outside of clinical trials may be systematically different from those enrolled in trials, and they may also receive different monitoring by their clinicians. Additional cases of rheumatic IRAEs have been seen by our group, but could not be included in this series due to clinical trial embargoes and publication restrictions.

There are many unanswered questions in the field of IRAEs due to ICIs. The diversity of manifestations of inflammatory arthritis, from type of joints involved, presence or absence of autoantibodies and presence or absence of erosive disease, demonstrates the need for careful baseline evaluation and following of these patients by rheumatologists. The complex phenotypes will not be understood without carefully cataloguing these patients. In addition, more work is needed to establish the incidence of rheumatic IRAEs. The denominator of patients treated with ICIs should be carefully evaluated so that the frequency of rheumatic manifestations can be understood and the risk of these events can be appropriately presented to patients. Finally, the rheumatologist's decisions on treatment for rheumatic IRAEs should be decided in conjunction with treating oncologists. It is unclear which disease-modifying antirheumatic drugs are safest or most effective in these patients. Given the grave prognosis of metastatic cancer, there is a higher acceptance of risk in treating IRAEs, with the goal of continuing ICI therapy. An example is the common use of TNF antagonists to treat the colitis associated with ICI therapy. ${ }^{31}$ The optimum dosage of corticosteroids is also uncertain as some patients in our series required doses as high of $120 \mathrm{mg}$ of prednisone daily for relief of their symptoms, while others could be maintained on a much lower dose after an initial high dose. Collaboration between rheumatologists and oncologists will be instrumental to understand the spectrum of rheumatological IRAEs and their treatment.
Correction notice This article has been corrected since it was published Online First. The second author's first name has been corrected.

Contributors LCC and COB planned the manuscript. All authors contributed to the composition and revision of the manuscript. JA obtained the ultrasound images for the manuscript.

Funding This project was supported by Grant Number P30-AR053503 from the National Institute of Arthritis and Musculoskeletal and Skin Diseases. AAS is supported by grant K23-AR061439. LCC is supported through a Jerome L. Greene Foundation Scholar Award. Additional support was provided by a Jerome L. Greene Foundation Discovery Award to COB.

Competing interests EJL, ANB and COB have served as consultants for Bristol-Myers Squibb. JRB has served as an unpaid consultant to Bristol-Myers Squibb. EJL also served as a consultant to Amgen and Merck. EJL has received research support from Bristol-Myers Squibb, Merck and AstraZeneca. JN has served as a consultant for Bristol-Myers Squibb and has received honoraria from Bristol-Myers Squibb and AstraZeneca. JRB and DL have received research funding from Bristol-Myers Squibb. Bristol-Myers Squibb provided no financial or writing support for this manuscript, but reviewed the manuscript for accuracy concerning patients who participated in clinical trials.

Provenance and peer review Not commissioned; externally peer reviewed.

\section{REFERENCES}

1 Lee L, Gupta M, Sahasranaman S. Immune Checkpoint inhibitors: an introduction to the next-generation cancer immunotherapy. J Clin Pharmacol 2016;56:157-69.

2 Weber JS, D'Angelo SP, Minor D, et al. Nivolumab versus chemotherapy in patients with advanced melanoma who progressed after anti-CTLA-4 treatment (CheckMate 037): a randomised, controlled, open-label, phase 3 trial. Lancet Oncol 2015;16:375-84.

3 Robert C, Schachter J, Long GV, et al. Pembrolizumab versus ipilimumab in advanced melanoma. N Eng J Med 2015;372:2521-32.

4 Larkin J, Hodi FS, Wolchok JD. Combined nivolumab and ipilimumab or monotherapy in untreated melanoma. N Engl J Med 2015;373:1270-1.

5 Hodi FS, O'Day SJ, McDermott DF, et al. Improved survival with ipilimumab in patients with metastatic melanoma. N Engl J Med 2010;363:711-23.

6 Garon EB, Rizvi NA, Hui R, et al. Pembrolizumab for the treatment of non-small-cell lung cancer. N Engl J Med 2015;372:2018-28.

7 Motzer RJ, Escudier B, McDermott DF, et al. Nivolumab versus everolimus in advanced renal-cell carcinoma. N Engl J Med 2015;373:1803-13.

8 Ansell SM, Lesokhin AM, Borrello I, et al. PD-1 blockade with nivolumab in relapsed or refractory Hodgkin's lymphoma. N Engl J Med 2015;372:311-19.

9 Topalian SL, Drake CG, Pardoll DM. Immune checkpoint blockade: a common denominator approach to cancer therapy. Cancer Cell 2015;27:450-61.

10 ClinialTrials.gov website: US National Institutes of Health. 2015 (accessed 27 Dec 2015).

11 Naidoo J, Page DB, Li BT, et al. Toxicities of the anti-PD-1 and anti-PD-L1 immune checkpoint antibodies. Ann Oncol 2015;26:2375-91.

12 Weber JS, Yang JC, Atkins MB, et al. Toxicities of Immunotherapy for the practitioner. J Clin Oncol 2015;33:2092-9.

13 Eisenhauer EA, Therasse P, Bogaerts J, et al. New response evaluation criteria in solid tumours: revised RECIST guideline (version 1.1). Eur J Cancer 2009;45:228-47.

14 Theander E, Mandl T. Primary Sjögren's syndrome: diagnostic and prognostic value of salivary gland ultrasonography using a simplified scoring system. Arthritis Care Res (Hoboken) 2014:66:1102-7.

15 Brahmer J, Reckamp KL, Baas $\mathrm{P}$, et al. Nivolumab versus docetaxel in advanced squamous-cell non-small-cell lung cancer. N Engl J Med 2015;373:123-35.

16 Robert C, Long GV, Brady B, et al. Nivolumab in previously untreated melanoma without BRAF mutation. N Engl J Med 2015;372:320-30.

17 Wolchok JD, Neyns B, Linette $G$, et al. Ipilimumab monotherapy in patients with pretreated advanced melanoma: a randomised, double-blind, multicentre, phase 2, dose-ranging study. Lancet Oncol 2010;11:155-64.

18 Bernardo SG, Moskalenko M, Pan M, et al. Elevated rates of transaminitis during ipilimumab therapy for metastatic melanoma. Melanoma Res 2013;23:47-54.

19 Topalian SL, Sznol M, McDermott DF, et al. Survival, durable tumor remission, and long-term safety in patients with advanced melanoma receiving nivolumab. J Clin Oncol 2014;32:1020-30.

20 Postow MA. Managing immune checkpoint-blocking antibody side effects. Am Soc Clin Oncol Educ Book 2015;35:76-83.

21 Borghaei $\mathrm{H}$, Paz-Ares L, Horn L, et al. Nivolumab versus docetaxel in advanced nonsquamous non-small-cell lung cancer. N Engl J Med 2015;373:1627-39.

22 Larkin J, Chiarion-Sileni V, Gonzalez R, et al. Combined nivolumab and ipilimumab or monotherapy in untreated melanoma. N Engl J Med 2015;373:23-34.

23 Chan MM, Kefford RF, Carlino M, et al. Arthritis and tenosynovitis associated with the anti-PD1 antibody pembrolizumab in metastatic melanoma. J Immunother 2015;38:37-9.

24 Bronstein $\mathrm{Y}, \mathrm{Ng}$ CS, Hwu P, et al. Radiologic manifestations of immune-related adverse events in patients with metastatic melanoma undergoing anti-CTLA-4 antibody therapy. AJR Am J Roentgenol 2011;197:W992-1000. 


\section{Clinical and epidemiological research}

25 Dulos J, Carven GJ, van Boxtel SJ, et al. PD-1 blockade augments Th1 and Th17 and suppresses Th2 responses in peripheral blood from patients with prostate and advanced melanoma cancer. J Immunother 2012;35:169-78.

26 Sheik Ali S, Goddard AL, Luke JJ, et al. Drug-associated dermatomyositis following ipilimumab therapy: a novel immune-mediated adverse event associated with cytotoxic T-lymphocyte antigen 4 blockade. JAMA Dermatol 2015;151:195-9.

27 Yoshioka M, Kambe N, Yamamoto Y, et al. Case of respiratory discomfort due to myositis after administration of nivolumab. J Dermatol 2015;42: 1008-9.
28 Goldstein BL, Gedmintas L, Todd DJ. Drug-associated polymyalgia rheumatica/giant cell arteritis occurring in two patients after treatment with ipilimumab, an antagonist of ctla-4. Arthritis Rheum 2014;66:768-9.

29 Weber JS, Kähler KC, Hauschild A. Management of immune-related adverse events and kinetics of response with ipilimumab. J Clin Oncol 2012;30:2691-7.

30 Woodworth T, Furst DE, Alten R, et al. Standardizing assessment and reporting of adverse effects in rheumatology clinical trials II: the Rheumatology Common Toxicity Criteria v.2.0. J Rheumatol 2007:34:1401-14.

31 Klair JS, Girotra M, Hutchins LF, et al. Ipilimumab-induced gastrointestinal toxicities: a management algorithm. Dig Dis Sci 2016. [Epub ahead of print]. 